\title{
Proteomic patterns of nipple aspirate fluids obtained by SELDI-TOF: Potential for new biomarkers to aid in the diagnosis of breast
}

\section{cancer}

\author{
Cloud P. Paweletz ${ }^{\mathrm{a}, \mathrm{b}, \mathrm{c}}$, Bruce Trock ${ }^{\mathrm{d}}$, \\ Marie Pennanen ${ }^{\mathrm{e}}$, Theodore Tsangaris ${ }^{\mathrm{e}}$, \\ Collette Magnant ${ }^{\mathrm{f}}$, Lance A. Liotta ${ }^{\mathrm{b}}$ and \\ Emanuel F. Petricoin III ${ }^{\mathrm{a}, *}$ \\ ${ }^{\mathrm{a}}$ Tissue Proteomics Unit, Division of Therapeutic \\ Proteins, CBER, Food and Drug Administration, \\ Bethesda, MD 20892, USA \\ ${ }^{\mathrm{b}}$ Laboratory of Pathology, National Cancer Institute, \\ National Institutes of Health, Bethesda, MD 20892, \\ USA \\ ${ }^{\mathrm{c}}$ Department of Chemistry, Georgetown University, \\ Washington, DC 20057, USA \\ ${ }^{\mathrm{d}}$ Department of Urology, Johns Hopkins University, \\ Baltimore, MD 21287, USA \\ ${ }^{\mathrm{e}}$ Department of Surgery, Georgetown University, \\ Washington, DC 20007, USA \\ ${ }^{\mathrm{f}}$ Department of Surgery, Sibley Hospital, Washington, \\ DC 20010, USA
}

\footnotetext{
Nipple aspirate fluid (NAF) has been used for many years as a potential non-invasive method to identify markers for breast cancer risk or early detection. Because individual markers have not been optimal, we are exploring the use of surface enhanced laser desorption and ionization time of flight (SELDITOF) mass spectrometry to identify patterns of proteins that might define a proteomic signature for breast cancer. SELDITOF was used to analyze a study set of NAF samples that included 12 women with breast cancer and 15 healthy controls (the latter included three women with an abnormal mammogram but subsequent normal biopsy). In this preliminary report, we present data showing that SELDI analysis of NAF is rapid, reproducible, and capable of identifying protein signatures that appear to differentiate NAF samples from breast cancer patients and healthy controls, including those with an abnormal mammogram who were later proven to be biopsy normal.
}

\section{Introduction}

Mammographic screening for breast cancer is currently the best available approach for early detection in the general population. However, additional approaches are needed. Current mammography is associated with a sensitivity of $75 \%-90 \%$ [1], but the positive predictive value at $25 \%$ is very low $[2,3]$. Although resolution continues to improve, mammography is still dependent on the existence of mass lesions. Because many breast tumors will already have metastasized by the time a mass is detectable [4], a significant portion of mammographically detected tumors in women undergoing regular screening will already be disseminated and incurable. Furthermore, mammography alone may not be sufficient for early detection in premenopausal women, particularly for young women with inherited susceptibility or other high risk profiles because of following reasons: (a) effectiveness of mammography has not been established in women younger than 40 , and is controversial in women aged 40-49, (b) younger women have more dense breast tissue, which reduces mammographic sensitivity, (c) tumor growth rates may be higher in younger women, and (d) women carriers of some germ-line mutations such as ataxia telangiectasia and possibly BRCA1/2 may have increased sensitivity to radiation and conceivably could be harmed by frequent mammograms [5-7].

More widespread testing of young women for germline predisposing mutations (such as BRCA1/2) will soon result in thousands of young women at high risk for breast cancer, for whom conventional screening approaches may be inadequate. Thus, there is an

${ }^{*}$ Corresponding author. Tel.: +1 301827 1753; Fax: +1 301480 3256; E-mail: petricoin@cber.fda.gov. 


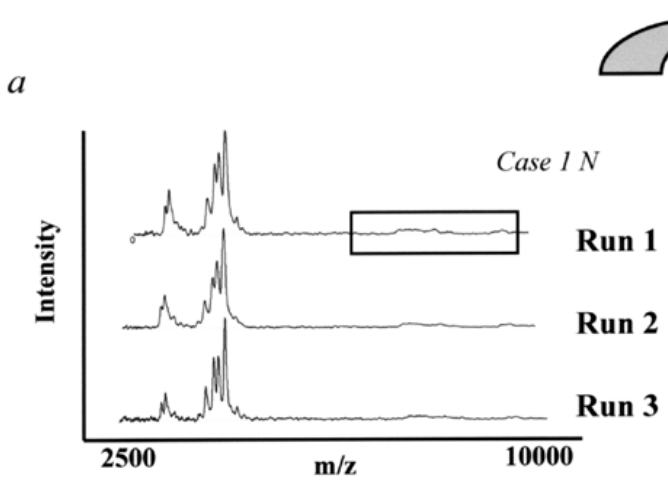

$b$

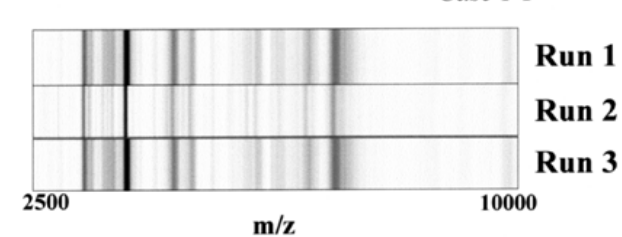

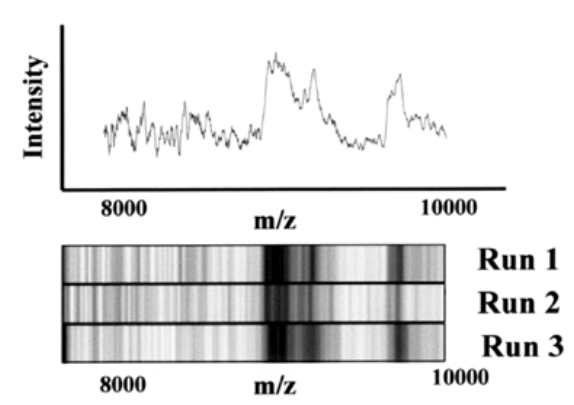

Case $2 T$

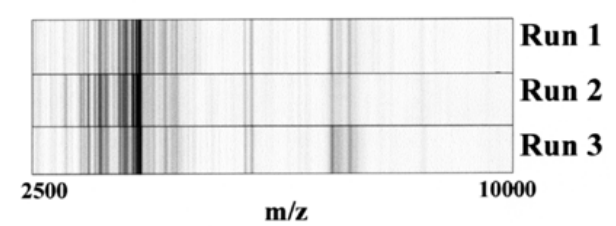

Fig. 1. SELDI-TOF spectra of nipple aspirate fluids are reproducible. a) Three representative SELDI-TOF chromatograms between 2500 Da and $10000 \mathrm{Da}$ from one case are shown. Good reproducibility between individually runs is achieved. Enlargement of mass ranges of proteins between $8000 \mathrm{Da}$ and $10000 \mathrm{Da}$ reveal additional individually resolved proteins that are reproducible. Chromatograms can be changed to density maps that represent the spectra as a gel view with each peak being a differently 'stained' band whose density is proportional to its height. b) Reproducibility of two different cases.

urgent need for additional methods of early detection that can provide an adjunct to mammography.

An alternative to imaging technologies for breast cancer detection is to examine easily accessible biological fluids for evidence of molecular signatures associated with neoplastic changes. Ideally such an approach should reflect the relevant biology of breast epithelium, be relatively non-invasive, easy for the patient, and conducive to serial monitoring. Nipple aspirate fluid (NAF) may be promising for direct sampling of breast epithelial biology. This fluid is secreted continuously by the non-lactating breast and can be aspirated through duct openings in the nipple using a simple non-invasive pump. Despite many years of study, however, no single marker in NAF has been shown to have high sensitivity and specificity for breast cancer [8-14].

Most molecular based approaches that have been investigated for early detection are targeted at specific defects, such as oncogenes, tumor suppressor genes, growth factors, tumor antigens, or other gene products. The inherent problem is that none of these factors alone are present in a large majority of breast cancers, and some are not specific to cancer or to breast tissue, so the sensitivity and specificity of such approaches is low. Rather than targeting a specific abnormality that may only be present in a small subgroup of patients, we propose to identify general patterns of protein expression that distinguish the invasive phenotype from preinvasive or normal tissue. Because such patterns could represent pathways commonly altered during neoplastic progression, it is highly likely that multiple elements of the pathway would be present in a majority of breast tumors, even if some individual protein alterations vary from case to case.

Surface-enhanced laser desorbtion time of flight spectrometry (SELDI-TOF) is a highly sensitive, specific, and high throughput technology platform for the study of whole protein lysates [15]. Protein lysates are directly applied onto a chromatographic surface (i.e. hydrophobic, hydrophilic, cationic, or anionic), washed, and subsequently analyzed by time of flight mass spectrometry. Thus, the obtained chromatogram only presents a protein phenotype of shared common chemical or physical characteristics. SELDI-TOF differs from conventional MALDI-TOF spectrometry in that it does not require initial separation of complex biological mixtures by high performance liquid chromatography (HPLC) or gas chromatography (GC) [16, 17]. The speed and ease of SELDI-TOF makes this technology very adaptable for use in the clinical laboratory. 


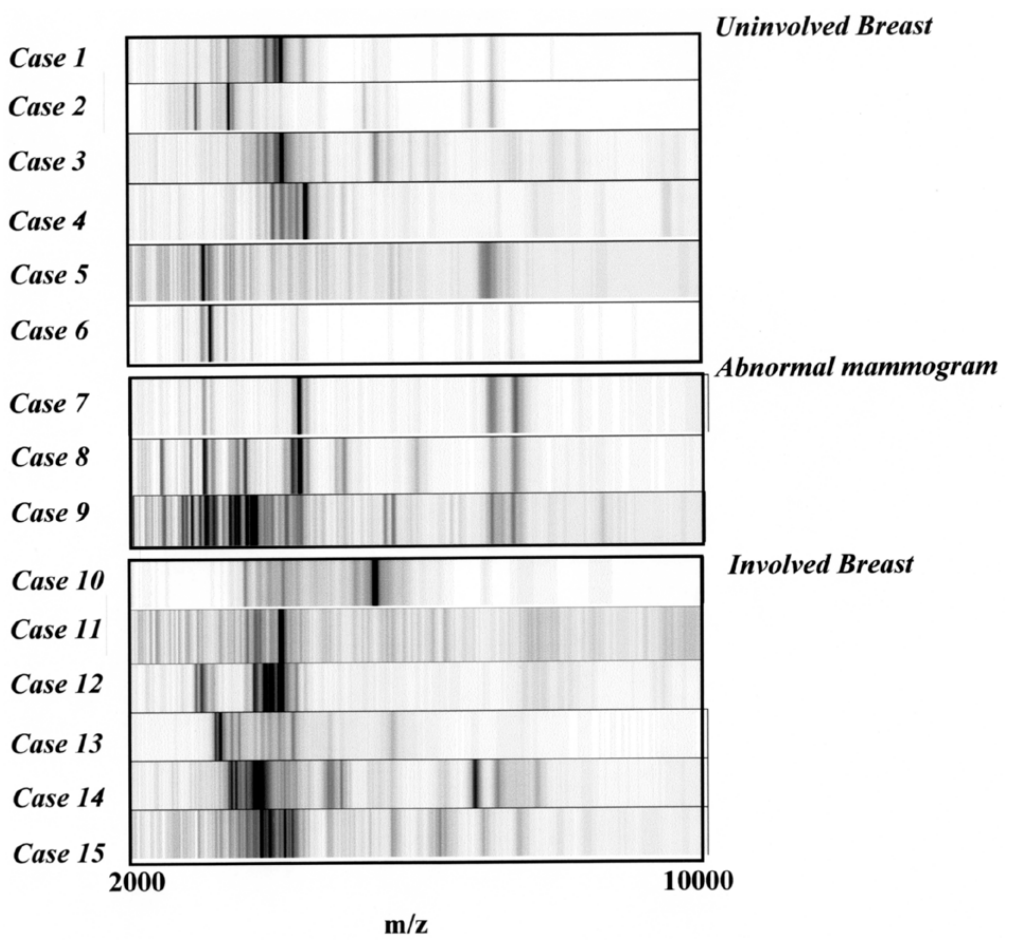

Fig. 2. Nipple aspirate fluids are marked by a high degree of heterogeneity. Representative gel view images of nipple aspirate fluids of three patients that were biopsy proven to be normal, but showed abnormal mammograms, six uninvolved breasts, and six involved breast are shown.

In this report, we used SELDI-TOF to generate protein profiles from NAF's of 27 patients ( 12 women with breast cancer and 15 healthy women; the latter included three women with repeated abnormal mammograms for whom subsequent biopsies were normal) to test the hypothesis that there exist low molecular weight proteomic signatures that can potentially distinguish breast cancer patients from healthy individuals.

\section{Patients and methods}

Study participants came from three sources: the Lombardi Cancer Center's Comprehensive Breast Center, the Georgetown University Breast Surgery clinic, and the office of a breast surgeon in private practice (CM). NAF was obtained using well-established methods [18] that we have successfully employed in previous studies. A modified breast pump is used, comprised of a finely polished glass cup with a Luer-lok end attached directly to a $20 \mu \mathrm{L}$ slip-tip syringe. The cup is placed over the cleansed nipple of the breast, and the woman compresses her breast with both hands while the plunger of the syringe is withdrawn to $10 \mu \mathrm{L}$ and held for 8-10 seconds. With this technique we have ob- tained NAF from 50-70\% of premenopausal women. Droplets of fluid that appear at any duct openings on the nipple are collected into capillary tubes. Three attempts of 8-10 seconds each were made to obtain fluid; if no fluid appears after three attempts the woman was considered a non-secretor. All fluid from an individual breast was pooled and expelled into $100 \mu \mathrm{L}$ of PBS. Samples from each breast were stored separately at $-70^{\circ} \mathrm{C}$ prior to analysis.

SELDI-TOF spectra were obtained as previously described [15]. Briefly, $1 \mu \mathrm{L}$ of acetonitrile was added onto a C-16 aliphatic chip (Ciphergen, Palo Alto, CA) followed by $1 \mu \mathrm{L}$ of NAF. The specimen was allowed to incubate under a humid atmosphere for at least 5 minutes after which the chip was thoroughly washed with water. The chip was air dried and $2 * 0.5 \mu \mathrm{L}$ of 3,5-dihydroxy cinnamic acid (diluted in 50\% Acetonitrile, $0.5 \%$ TFA) was co-crystallized. The instrument was operated in linear mode. Three profiles totaling 35 shots were obtained from 3 of the 100 addressable regions for a given spectrum. All multiparametric analyses were performed using PEAKS 2.0.1 (Ciphergen, Palo Alto, CA). 


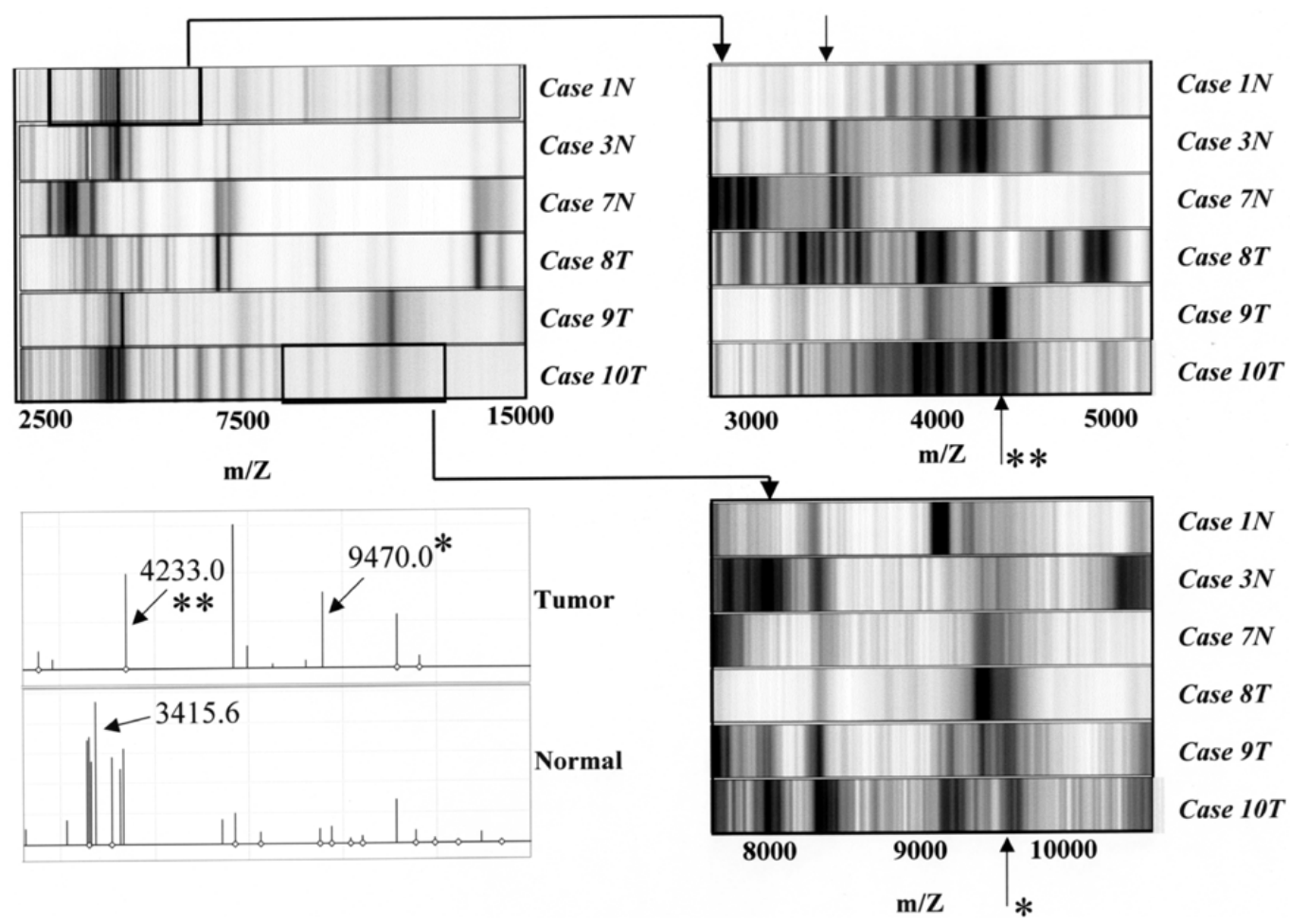

Fig. 3. Generation of Normal and Tumor specific tumor plates. a) SELDI-TOF spectra from 12 tumor and 15 normal NAF samples were run (in triplicates, with three representative GelViewTM spectra shown) and compared to each other. Proteins that were within $0.75 \%$ of each other were considered to be the same molecular identity and thereby placed into the protein template. Protein identities that were not present in all analyzed cases were deemed not suitable for diagnosis purposes. Four proteins were present in NAFs of normal and cancerous breasts at 3415.6 Da and 4149.7 Da, as well as 4233.09 $\mathrm{Da}$ and 9470.0 Da, respectively.

\section{Results}

Previous experiments showed that body fluids such as saliva (unpublished data) and serum (manuscript submitted) are compatible with SELDI-TOF and yield very reproducible protein patterns under $20 \mathrm{kDa}$ without the need to purify the samples by HPLC or size exclusion chromatography. These results are in line with previously published reports that show that MALDITOF spectra of serum are richer in ion species in molecular mass ranges that are smaller than $30 \mathrm{kDa}[15,19]$. While protein patterns of NAF's at higher molecular weights (up to $150 \mathrm{kDa}$ ) can easily be obtained the diagnostic utility diminishes rapidly due to well known ion suppression effects (data not shown). Coefficients of variances for inter and intra runs were previously shown to be less than $10 \%$ [15]. Concurrently, using an aliphatic $\mathrm{C} 16$ chip yields very reproducible spectra between $2 \mathrm{kDa}$ and $15 \mathrm{kDa}$ for NAFs with an average of over 50 individually resolved proteins in the mass range. Each sample was run in triplicates to ensure that proteins seen during individual runs were indeed reproducible (Fig. 1). Day to day or instrument variations can be excluded as samples run on different days yielded identical spectra (data not shown).

However, in contrast to serum and saliva chromatograms that do not show large variations (data not shown), NAFs, while being reproducible, are marked by unusual large variations in their spectra between different samples within a group (Fig. 2). Confronted with this observation, we decided against measuring individual protein abundance levels, but rather decided to generate a protein template that presents only the proteins that are consistently associated with cancerous or normal breasts. This was achieved by comparing all proteins and peptides that are greater than two standard deviations above background of all cancerous breasts and all normal NAF specimens, respectively. Proteins that were within $0.75 \%$ mass accuracy of each other were considered the same and thus compared with the next respective sample. A protein profile for normal and tumor NAF samples is shown in Fig. 3. The dramatic reduction in proteins compared to those detected without the above criteria i.e. from more than 50 pro- 
a)

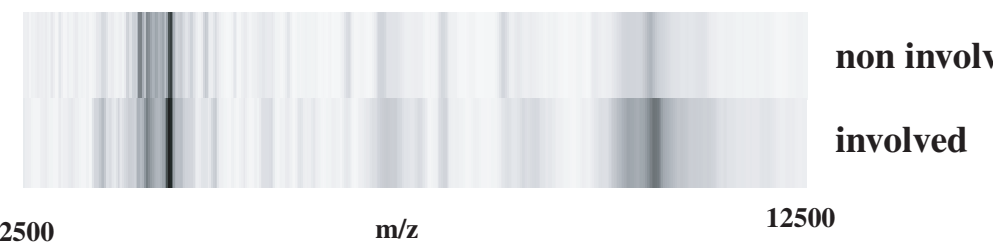

b)

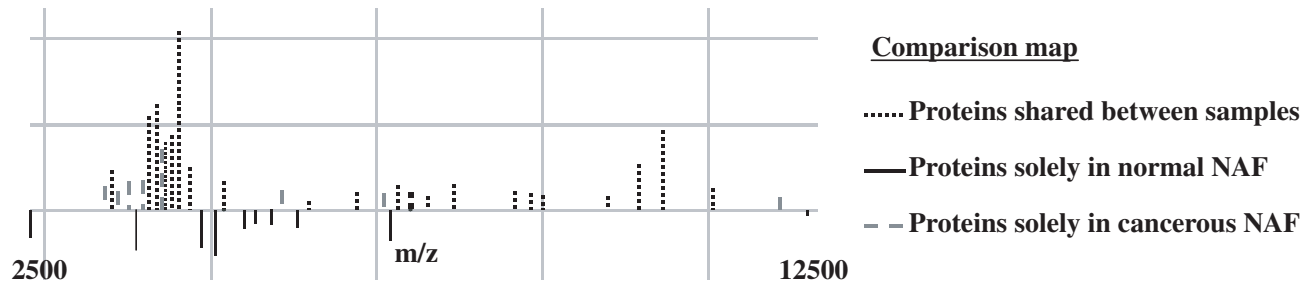

c)

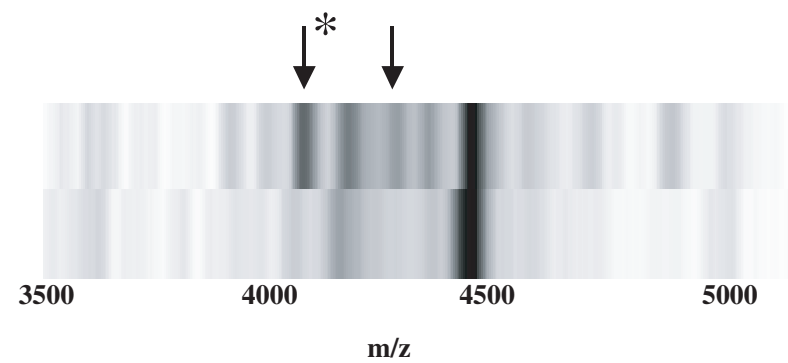

\section{non involved}

involved

Fig. 4. Protein Profiles from patient matched NAFs from involved an uninvolved breasts. SELDI spectra were taken between 2500 Da and $15000 \mathrm{Da}$ of patient matched NAFs with one of the breast being biopsy proven cancerous, and the other being normal. As expected, most of the proteins did not change, however protein changes track with generated template (arrow).

teins to 10 or fewer) is indicative of the large heterogeneous nature of these samples with respect to protein expression. However, two proteins were uniquely present in tumor-associated samples (at $4233.09 \mathrm{~m} / \mathrm{z}$ and $9470.0 \mathrm{~m} / \mathrm{z}$ ) and two were uniquely associated with normal samples (at $3415.6 \mathrm{~m} / \mathrm{z}$ and $4149.7 \mathrm{~m} / \mathrm{z}$ ).

This template was tested in a variety of cases for whom NAFs were obtained from both the cancerous and contralateral breast (Fig. 4). As expected most proteins do not change between the involved and the contralateral uninvolved breast and appear very different from the generated templates; however, the individual identified proteins always tracked with the disease state.

A powerful application of this technology would be as an adjunct to improve specificity of the diagnostic paradigm in women with abnormal mammograms. A protein template of NAFs from three women with abnormal mammograms was generated, and protein templates from the cancerous and normal breasts were compared (Fig. 5). All three of these women were biopsied and found to have normal breasts. The chromatogram obtained from women with abnormal mammograms is distinct from that of both the normal and tumorassociated specimens. However, the protein identity at 4152.3 Da that is present in normal SELDI-TOF template, but not in the cancerous template, can be found. As the mass accuracy of the machine is between $0.75 \%$ and $1 \%$ the protein identities at $4152.3 \mathrm{Da}$ and 4149.7 Da are likely to be the same. Furthermore, each protein identity that is present in the tumor template is not present in the abnormal mammogram spectra (Fig. 5). These results indicate that spectra obtained by SELDI may aid in the decision making process for patients that show an abnormal mammogram.

\section{Discussion}

The need for rapid, inexpensive, noninvasive approaches to improve the specificity of breast cancer screening paradigms has led to the search for tumor markers in readily accessible body fluids. However, the search for such individual markers have been dis- 


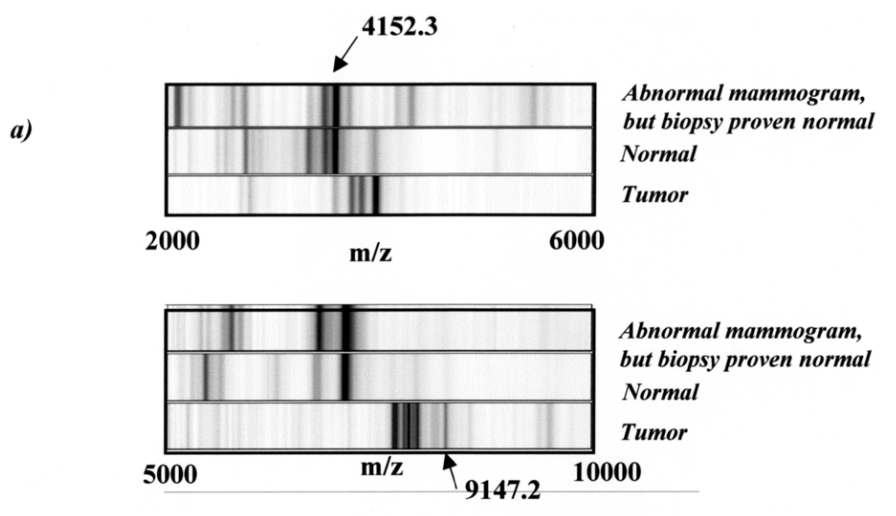

b)

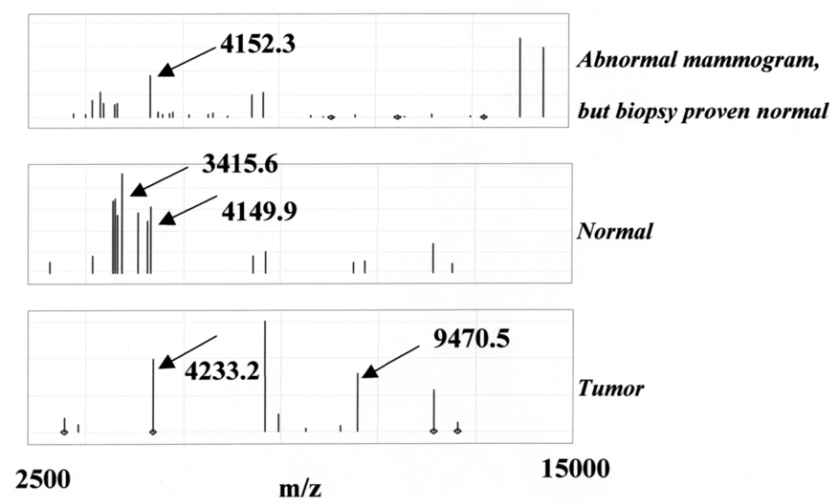

Fig. 5. SELDI analysis of NAFs from biopsy proven normal patients with abnormal mammograms. SELDI templates of three patients that were biopsy proven to be normal, but showed abnormal mammograms were compared to the normal and cancerous template. While providing an unique SELDI protein pattern in itself tumor unique proteins (4233.09 Da and 9470.0 Da) were absent, and proteins that were specific to normal samples (4197.9 Da) were present in NAFs of breasts that showed abnormal mammograms.

appointing [20,21]. We [15] and others [16,22,23] are utilizing rapid proteomic approaches that can easily be implicated to the clinic and aid in the diagnosis decision making. Toward this end, our laboratory currently employs two approaches utilizing affinity SELDI-TOF to identify breast cancer protein signatures in NAF and serum. Such a signature will be a pattern of protein expression that consistently is associated with cancer and discriminates it from samples from women with normal breasts. The simplest approach to identifying such a signature is based on identifying low molecular weight proteins and peptides $(<10,000 \mathrm{Da})$ that are present in either $100 \%$ of tumor-associated or normalassociated samples. The discovery of multiple proteins and patterns of protein expression that are consistently altered may increase the specificity and sensitivity of an eventual diagnosis.

Here, we report the use of SELDI-TOF to generate protein profiles from NAF's obtained from 27 women (12 with breast cancer and 15 without breast cancer). This study group also included three women with ab- normal mammograms but found to be normal at biopsy. Two peptides at 4233.09 Da and 9470.0 Da were consistently associated with tumor samples, while two peptides at 3415.6 Da and 4149.7 Da consistently appeared in normal samples, and thus may be of diagnostic importance. Moreover, overall protein patterns obtained from NAFs from women with abnormal mammograms did not clearly resemble protein patterns from normal or tumor patients. However, all three cases contained a 4152.3 Da that showed resemblence to the 4149.9 Da. Furthermore, no proteins that are unique to tumor samples were found in the abnormal cases.

While matrix assisted laser desorption spectrometry (MALDI-TOF) has been used to analyze known tumor markers these studies are not really amenable to clinical settings $[23,24]$. The speed and ease of SELDI-TOF to generate protein patterns without the need to purify the specimen prior to application makes this technology very adaptable for use in the clinical laboratory. We believe that advances in this technology will allow 
clinical investigators to monitor proteomic patterns for diagnosis or prognosis.

\section{References}

[1] W.L. Donegan, Evaluation of a palpable breast mass, $N$ Engl J Med 327 (1992), 937-942.

[2] J.R. Harris, M.E. Lippman, U. Veronesi and W. Willett, Breast cancer, N Engl J Med 327 (1992), 319-328.

[3] J.G. Elmore, M.B. Barton, V.M. Moceri, S. Polk, P.J. Arena and S.W. Fletcher, Ten-year risk of false positive screening mammograms and clinical breast examinations, $N$ Engl J Med 338 (1998), 1089-1096.

[4] B. Fisher, The evolution of paradigms for the management of breast cancer: a personal perspective, Cancer Res 52 (1992), 2371-2383.

[5] M. Swift, Ionizing radiation, breast cancer, and ataxia_telangiectasia, J Natl Cancer Inst 86 (1994), 15711572 .

[6] S.K. Sharan, M. Morimatsu and U. Albrecht et al., Embryonic lethality and radiation hypersensitivity mediated by Rad51 in mice lacking Brca2, Nature 386 (1997), 804-810.

[7] R. Scully, J. Chen and R.L. Ochs et al., Dynamic changes of BRCA1 subnuclear location and phosphorylation state are initiated by DNA damage, Cell 90 (1997), 425-435.

[8] M. Wrensch and N.L. Petrakis et al., Breast cancer risk associated with abnormal cytology in nipple aspirates of breast fluid and prior history of breast biopsy, Am J Epidemiol 137 (1993), 829-833.

[9] E.R. Sauter, E. Ross and M. Daley et al., Nipple aspirate fluid: A promising non-invasive method to identify cellular markers of breast cancer risk, Br J Cancer 76 (1997), 494-501.

[10] Y. Liu, J.L. Wang, H. Chang, S.H. Barsky and M. Nguyen, Breast-cancer diagnosis with nipple fluid bFGF, Lancet 356 (2000), 567.

[11] Y.Zhao, J.V. Sigitas, N. Klar, N.L. Sadowsky, C.M. Kaelin and B. Smith, Nipple fluid carcinoembryonic antigen and prostate antigen in cancer bearing and tumor-free breasts, J Clin Oncol 19 (2001), 1462-1467.

[12] E.R. Sauter, M. Daly and K. Linahan et al., Prostate Specific antigen levels In nipple aspirate fluid correlate with breast cancer risk, Cancer Epideminol Biomarkers Prev 5 (1996), 967-970.
[13] E.B. King, K.L. Chew, N.L. Petrakis and V.L. Ernster, Nipple apirate cytology for the study of breast cancer precursor, $J$ Natl Cancer Inst 71 (1983), 1115-1121.

[14] L. Foretova, J. Garber and N. Sadowsky et al., Carcinoembryonic antigen in breast nipple spirate fluid, Cancer Epidemiol Biomarkers Prev 7 (1998), 195-198.

[15] C.P. Paweletz, J.W. Gillespie and D.K. Ornstein, Rapid protein display profiling of cancer progression directly from human tissue using a protein biochip, Drug Dev Res 49 (2000), 34-42.

[16] T. Nakanishi, A. Shimizu, N. Okamoto, A. Ingendoh and M. Kanai, Analysis of serum protein precipitated with antiserum by matrix-assisted laser desorption ionization/time of flight and electrospray ionization mass spectrometry as a clinical laboratory test, J Am Soc Mass Spectrom 6 (1995), 854-859.

[17] P. Chaurand, M. Stoeckli and R.M. Caprioli, Direct Profiling of proteins in biological tissue sections by MALDI mass spectrometry, Anal Chem 71 (1999), 5263-5270.

[18] N.L. Petrakis, ASO distinguished achievement award lecture. Studies on the epidemiology and natural history of benign breast disease and breast cancer using nipple aspirate fluid, Cancer Epidemiol Biomarkers Prev 2 (1993), 3-10.

[19] L. Ferrari, R. Seraglia, C.R. Rossi, A. Bertazzo, M. Lise and A. Graziella et al., Protein profiles in sera of patients with malignant cutaneous melanoma, Rapid Com in Mass Spec 14 (2000), 1149-1154.

[20] R.B. Dickson, J.A. Low, M.D. Johnson, M.J. Hawkins and B.J. Trock, Blood-borne indicators of breast cancer and their use in experimental, medical oncologic, and prevention studies, The Breast 5 (1996), 379-384.

[21] M.A. Harding and D. Theodorescu, Prognostic markers in localized prostate cancer: from microscopes to molecules, Cancer and Met Reviews 17 (1999), 429-437.

[22] J. Khan, J.S. Wei, M. Ringer, L.H. Saal, M. Ladanyi and F. Westermann et al., Classification and diagnostic prediction of cancers using gene expression profiling and artifical neural networks, Nature (2001), 673-679.

[23] J.M. Lacey, H.R. Bergen, M.J. Magera, S. Naylor and J.F. O'Brien, Rapid determination of transferrin isoforms by immunoaffinity liquid chromatography and electrospray mass spectrometry, Clin Chem 47 (2001), 513-518.

[24] A. Lapolla, D. Fedele, R. Aronica, M. Garbeglio, M. Alpaos, R. Seraglia and P. Traldi, Evaluation of IgG Glycation levels by matrix-assisted laser desorption/ionization mass spectrometry, Rapid Commun Mass Spectrom 11 (1997), 1342-1346. 


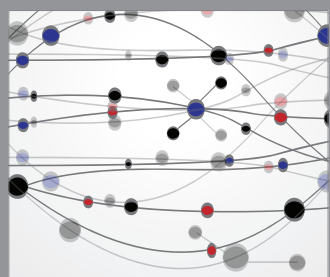

The Scientific World Journal
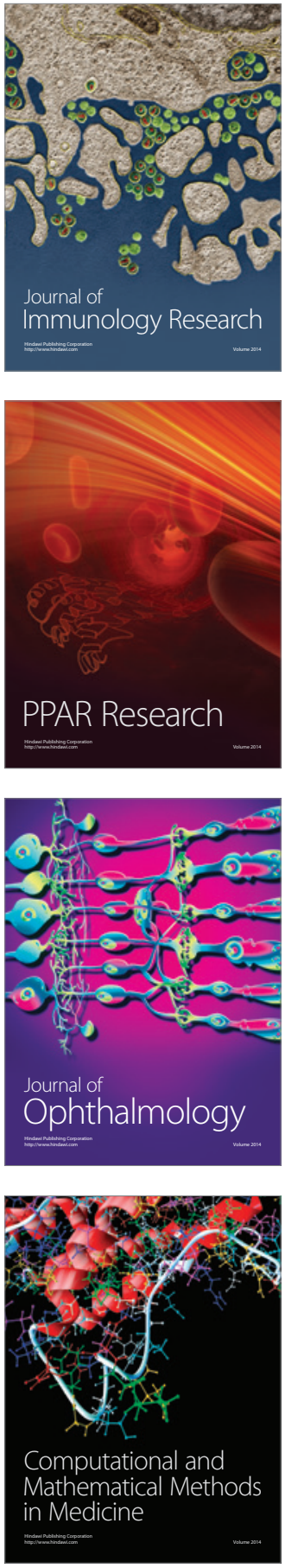

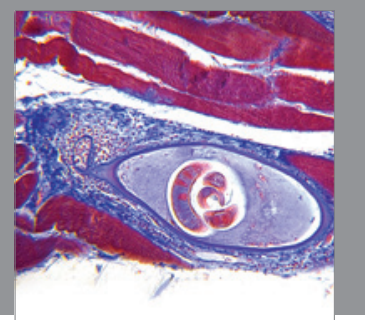

Gastroenterology

Research and Practice
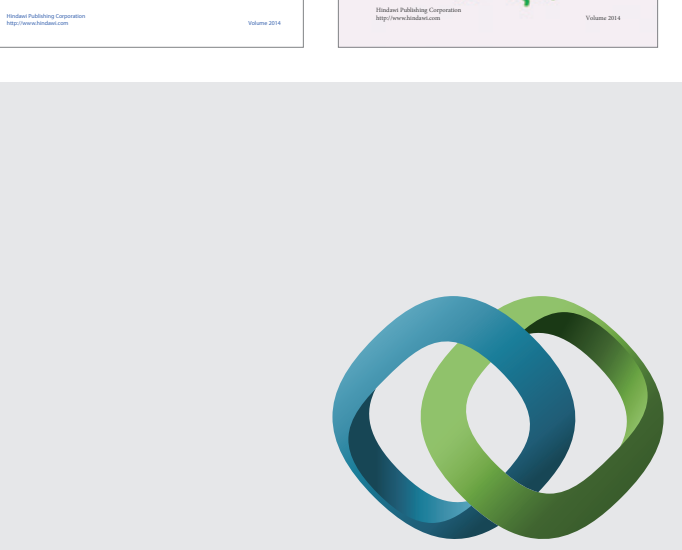

\section{Hindawi}

Submit your manuscripts at

http://www.hindawi.com
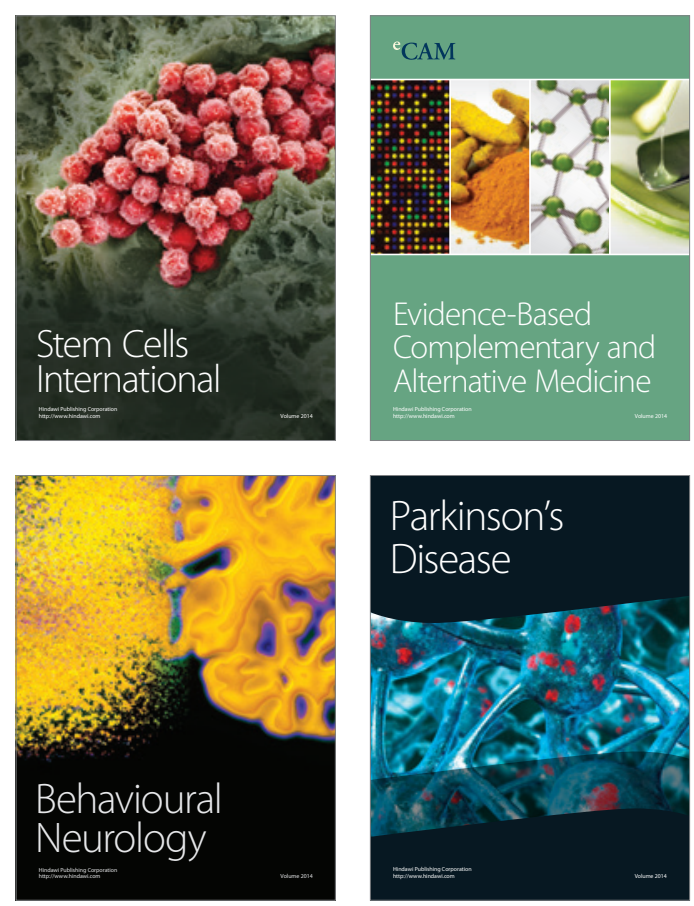

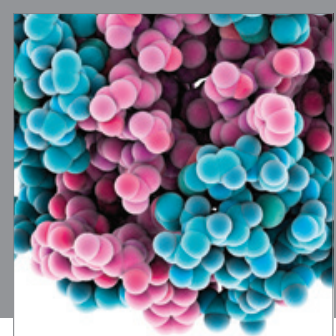

Journal of
Diabetes Research

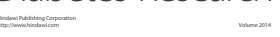

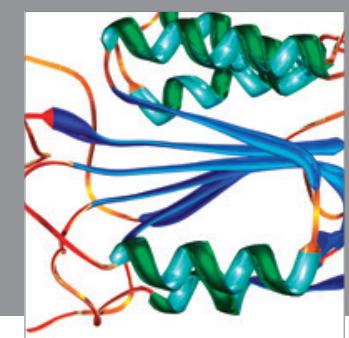

Disease Markers
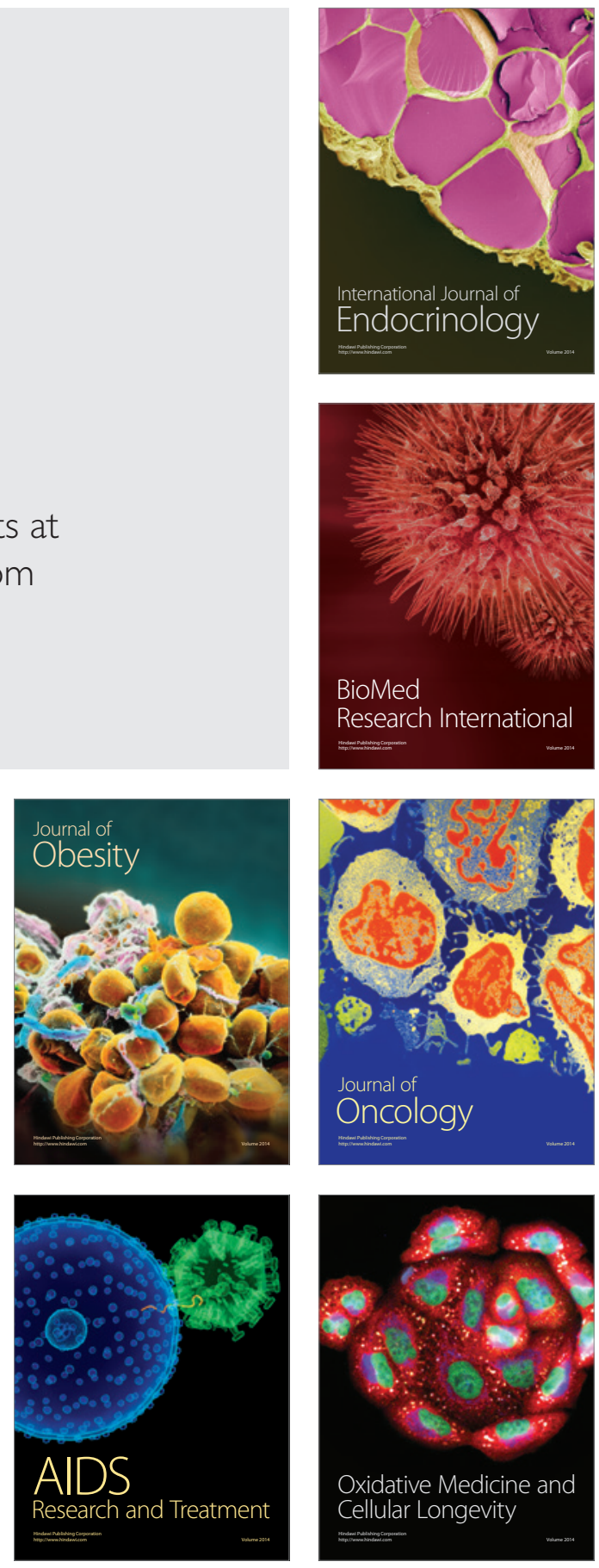